\title{
Boundary Layer Approximation for Melt Film Dynamics in Laser Fusion Cutting
}

\author{
$\underline{\text { U. Jansen }}^{\text {a }}$, M. Niessen ${ }^{\text {b }}$, T. Hermanns ${ }^{\mathrm{a}}$, D. Arntz ${ }^{\mathrm{c}}$, R. Poprawe ${ }^{\mathrm{cb}}$ and W. Schulz ${ }^{\mathrm{ab}}$ \\ ${ }^{a}$ RWTH Aachen University - Nonlinear Dynamics of Laser Processing, Steinbachstr. 15, 52074 Aachen, \\ Germany \\ ${ }^{\mathrm{b}}$ Fraunhofer Insititute for Laser Technology, Steinbachstr. 15, 52074 Aachen, Germany \\ ${ }^{\mathrm{c}}$ RWTH Aachen University - Chair for Laser Technology, Steinbachstr. 15, 52074 Aachen, Germany \\ Email: ulrich.jansen@nld.rwth-aachen.de
}

\begin{abstract}
During laser fusion cutting of sheet metal parts, a focused laser beam is traversed relatively to the processed part. The material is molten due to the heat input of the laser beam and driven out of the cutting kerf by a gas jet aligned coaxially with the laser. The edges of the remaining cutting kerf show a striation pattern which affects the quality of the cut. The amplitude, wavelength and shape of these striations depend on the dynamics of the motion of the thin melt film inside the interaction zone.

Instabilities in the thickness of the melt film that emerge at the cutting front propagate to the sides and solidify to an irregular surface. Understanding effects that lead to the emergence and stirring up of instabilities is crucial to derive measures for high quality cuts with a drastically reduced striation depth.

To simulate the behavior of the thin melt film, the underlying incompressible Navier Stokes equations have to be solved with high accuracy using a well chosen set of boundary conditions. The surface of the melt film moves with a velocity of up to several meters per second from top to bottom side while the thickness of the melt film varies from a few to around one hundred micro meters perpendicular to the laser beam. These scales prevent the use of classical finite element or finite volume approaches to solve the mathematical problem numerically accurate without proper reduction techniques.

To develop a simulation that is able to depict the behavior of the melt film, the Navier Stokes equations are transformed to conformal coordinates and subjected to scaling analysis. A perturbation series expansion is performed and the equations are integrated in radial direction using a quadratic ansatz for the mass flux in azimuthal and axial direction. The resulting system of partial differential equations can be solved numerically and describes significant properties of the dynamics of the melt film with high temporal and spatial resolution. The physical mechanisms that lead to striation formation can be investigated by analysis of the model structure as individual physical phenomena can be selectively altered in the simulation.

The presented method leads to a simulation that provides support in the evaluation of measures to reduce the striation depth like modulation of laser power or beam shaping optics. The presented combination of model reduction techniques is adaptable to any boundary layer problem of similar type.
\end{abstract}

Keywords: Boundary layer approximation, laser fusion cutting, incompressible Navier Stokes equation 


\section{INTRODUCTION}

In laser fusion cutting of sheet metals, quality reducing striations occur on the cutting kerf of the processed part, which cause a measurable roughness as shown in Figure 1. Experimental observation by Arntz et al. [2017] using the trim cut analysis show that these striations happen by solidification of small ligaments of the melt film in the wake of the cutting process. The formation of ligaments is caused by inherent instabilities that exist in laser cutting as shown by Vossen and Schüttler [2012]. To understand which processes and which parameters tend to increase or decrease the amplitude of these instabilities, modeling and simulation of the melt film dynamics carried out.

Otto and Schmidt [2010] present a full featured simulation of laser fusion cutting using the finite volume software OPENFOAM. Because of the complexity of the gas dynamic simulation to model the driving forces of the process gas flow, the resolution of the resulting simulation is limited by the computational effort that can be made for a full featured process simulation. Kohl and Schmidt [2013] reduce the effort by applying pressure boundary conditions for the molten phase and show the effect of different laser beam shapes on the behavior of the melt film. Kheloufi et al. [2015] use the computational fluid dynamics software FLUENT to model the laser cutting process and demonstrate that the effect of Fresnel absorption is important for the final shape of the cutting kerf. Amara et al. [2015] enhance this model by taking surface tension into account, which contributes to the formation of striations.

However, these full featured simulations of laser processing are limited by the computational effort that can be performed for an individual simulation run. Typical scales of laser fusion cutting include melt film thicknesses of several $10 \mu \mathrm{m}$ and melt flow velocities above $1 \mathrm{~m} \mathrm{~s}^{-1}$ [Wandera and Kujanpaa, 2010]. The resulting spatiotemporal scales to describe the dynamics of the thin melt film in laser fusion cutting demand for proper reduction techniques and adapted solution methods. An approach for a boundary layer approximation using conformal coordinates and a perturbation expansion is presented in this paper.

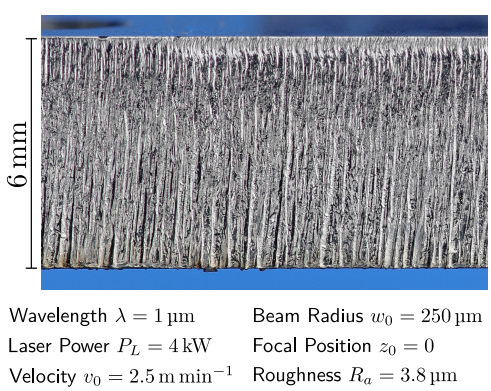

Figure 1. Striations in laser cutting. Especially for $1 \mu \mathrm{m}$ wavelength laser sources the processed part shows a certain roughness.

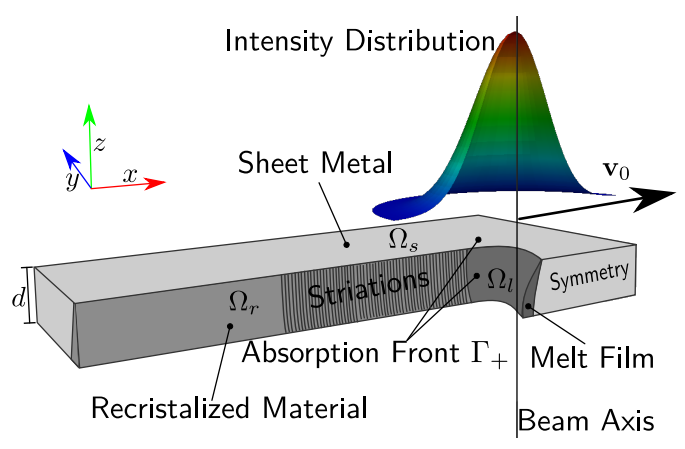

Figure 2. Sketch of laser fusion cutting. Perturbations in the solidifying melt film cause a striation pattern on the processed cutting kerf.

\subsection{Model Reduction and Scale Separation}

Langtangen et al. [2002] present several numerical solution schemes for incompressible viscous flows and give hints about the computational implications while solving these problems, including operator splitting techniques. For laser fusion cutting a hierarchy of spatial dimension can be identified as shown by Schulz et al. [1999]. A 2D-description of the melt using integral methods is given by Schulz et al. [2009]. Figures 2 and 3 depict the length scales present for the melt film. Conformal coordinates are presented in Figure 4 and natural length scales of the process can be identified. The axial $z$-direction perpendicular to the surface of the sheet metal and along with the laser beam shows the largest length scale represented by the sheet thickness $d$. The azimuthal scale of the lateral $\alpha$-direction is represented by the laser beam radius $w_{0}$ and the radial length scale in $\nu$-direction has a typical length scale of the melt film thickness $d_{m}$. For a typical laser fusion cutting task follows

$$
d_{m} \ll w_{0} \ll d
$$


These scales describe typical average values for the modeled process and are set constant during a specific computation. Because of the presence of this hierarchy of spatial dimensions in the conformal description of the melt film, the underlying equations are expanded to a perturbation series [Kato, 1995]. A power series expansion [Lopez-Sandoval and Mello, 2012] of the quantities velocity, pressure and temperature enables a hierarchical solution sequence that allows high accuracy at critical and reduced accuracy at smooth regions of the melt film. The resulting expression of the NAVIER-STOKES and energy equations is well suited for an integral boundary layer solution method that allows fast and accurate computational solutions. The general applicability of a perturbation analysis to describe the dynamics of the melt at the apex is given by Poprawe et al. [2010] using a 2D model based on the mathematical analysis presented by Vossen and Schüttler [2012].

\section{Mathematical TASK}

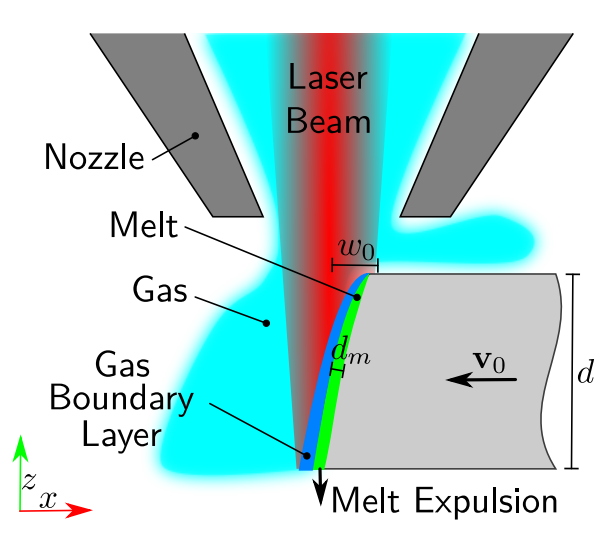

Figure 3. 2D sketch of laser fusion cutting including feed velocity $v_{0}$ and the typical length scales sheet thickness $d$, beam radius at focal position $w_{0}$ and av-erage melt film thickness $d_{m}$.

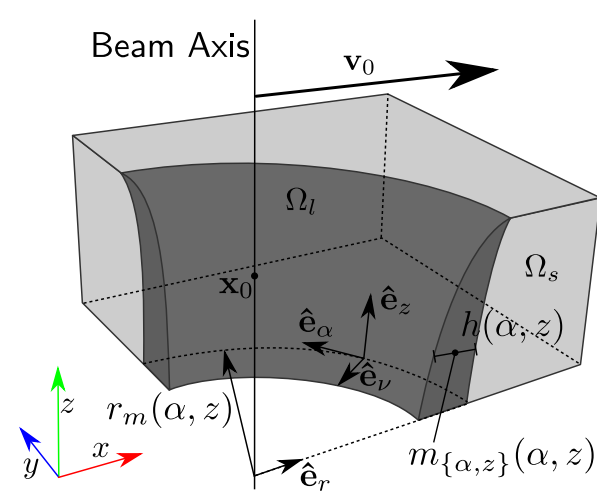

Figure 4. Conformal coordinates for the melt film in laser fusion cutting. The integral model solves for the characteristic variables melt film thickness $h$ and integrated mass fluxes in azimuthal $m_{\alpha}$ and axial $m_{z}$ directions.

The mathematical task of laser fusion cutting is presented by Schulz et al. [2009]. Details about the absorbed heat flux are given by Mahrle and Beyer [2009]. The set of equations that has to be solved for the melt film includes the balance of mass, the Cauchy momentum equation in conservative form neglecting the gravitational force and the heat flux equation as energy balance equation.

$$
\begin{aligned}
\nabla \mathbf{v} & =0 & & \text { MASS } \\
\partial_{t} \mathbf{v}+(\mathbf{v} \cdot \nabla) \mathbf{v} & =-\frac{1}{\varrho} \nabla p+\nu_{l} \Delta \mathbf{v} & & \text { MOMENTUM } \\
\partial_{t} T+(\mathbf{v} \cdot \nabla T) & =\kappa \Delta T & & \text { ENERGY }
\end{aligned}
$$

for the quantities flow velocity $\mathbf{v}=\mathbf{v}(\mathbf{x}, t)$, temperature $T=T(\mathbf{x}, t)$ and pressure $p=p(\mathbf{x}, t)$ for $\mathbf{x} \in \Omega_{l}(t)$ and the material parameters density $\varrho$, kinematic viscosity $\nu_{l}$ and thermal diffusivity $\kappa$. For a given heat flux $Q_{[s, l]}=k \nabla T_{[s, l]} \cdot \mathbf{n}$ for the solid and the liquid phase, an inflow $\mathbf{v}_{i n}$ and a fixed temperature at melting temperature $T_{m}$, the boundary conditions for the melting boundary $\Gamma_{m}$ are given as

$$
Q_{s}=Q_{l}-\varrho h_{m} v_{p}^{m}, \quad T=T_{m}, \quad \mathbf{v}=\mathbf{v}_{i n}(t), \quad \mathbf{x} \in \Gamma_{m}(t) .
$$

with specific melting enthalpy $h_{m}$ and normal velocity of the phase boundary $v_{p}^{m}$. At the absorption boundary $\Gamma_{a}$ no material is transfered in normal direction $\mathbf{n}$ and the normal velocity of the phase boundary is $v_{p}^{a}$. The process gas pressure $p_{g}$ and the Laplace pressure for the surface tension $\sigma$ and the curvature $\mathcal{K}$ act as load for the normal component of the stress tensor, the driving forces $\tau_{g[1,2]}$ and the thermal gradient of the surface tension act as load for the tangential components in the tangential directions $t_{[1,2]}$. The absorbed energy flux 
U. Jansen et al., Boundary layer approximation for melt film dynamics in laser fusion cutting

density $Q_{a}$ of the laser beam acts as load for the temperature $T$.

$$
\begin{aligned}
\rho\left(\mathbf{v}-v_{p}^{a} \mathbf{n}\right) \mathbf{n} & =0, & \mathbf{n}(\mathfrak{S} \mathbf{n}) & =-p_{g}+\sigma \mathcal{K}, \\
\mathbf{t}_{[1,2]}(\mathfrak{S} \mathbf{n}) & =\tau_{g[1,2]}-\frac{\mathrm{d} \sigma}{\mathrm{d} T}\left(\nabla T \cdot \mathbf{t}_{[1,2]}\right), & k \nabla T \cdot \mathbf{n} & =Q_{a}
\end{aligned}
$$

for $\mathbf{x} \in \Gamma_{a}(t)$ and the thermal conductivity $k$. The stress tensor $\mathfrak{S}$ for incompressible fluids is the sum of a hydrostatic and a viscous contribution

$$
\mathfrak{S}=-p \mathfrak{I}+\eta\left(\nabla \otimes \mathbf{v}+(\nabla \otimes \mathbf{v})^{T}\right)
$$

with the identity $\mathfrak{I}$, the dynamic viscosity $\eta$ and the dyad $\mathbf{a} \otimes \mathbf{b}=\mathbf{a b}^{T}$. At the bottom boundary $\Gamma_{b}$, a continuity boundary condition is applied to describe the outflow of the molten material.

The melt film thickness is described by a kinematic boundary condition. The surface of the melt film is given by the implicit function

$$
h(\mathbf{x}, t)=0, \quad \mathbf{n}=\nabla h, \quad \mathbf{x} \in \Gamma_{a}(t)
$$

By neglecting the evaporation mass flux, the normal velocity of the surface $h$ equals the normal velocity of the melt. Using the incompressibility $\nabla \cdot \mathbf{v}=0$ the evolution of the melt film thickness is given by

$$
\partial_{t} h+\mathbf{v} \cdot \nabla h=0
$$

\section{REduced Model}

To perform a scaling analysis on the equations for the melt film, the equations (2) are translated to conformal coordinates which create a coordinate system in the melt film. The transformed equations are subjected to a scaling analysis and expanded in a perturbation series. The final expressions are integrated into radial direction using a quadratic ansatz for the velocity $\mathbf{u}$. The resulting set of equations describes the integrated mass fluxes $m_{\alpha}, m_{z}$ and the thickness of the melt film $h$.

\subsection{Conformal Coordinates}

Figure 4 shows the coordinate system in the melt film which defines axial $(z)$, azimuthal $(\alpha)$ and radial $(\nu)$ components. The transformation to Cartesian coordinates with origin $\mathbf{x}_{0}$ on the laser beam axis and the top side of the part is given by

$$
\mathbf{x}(\nu, \alpha, z, t)=\mathbf{x}_{0}(t)+z \hat{\mathbf{e}}_{z}+r_{m}(\alpha, z, t) \hat{\mathbf{e}}_{r}+\nu \hat{\mathbf{e}}_{\nu}
$$

for the position of the melting front $r_{m}$ and the unit vectors $\hat{\mathbf{e}}$. To describe derivatives in the covariant coordinate system $\{\nu, \alpha, z\}$, the Lamé coefficients (also named scale factors)

$$
h_{\nu}=\left|\frac{\partial \mathbf{x}}{\partial \nu}\right|=1, \quad h_{\alpha}=\left|\frac{\partial \mathbf{x}}{\partial \alpha}\right|=\sqrt{\left(\nu-r_{m}\right)^{2}+\left(\partial_{\alpha} r_{m}\right)^{2}}, \quad h_{z}=\left|\frac{\partial \mathbf{x}}{\partial z}\right|=\sqrt{1+\left(\partial_{z} r_{m}\right)^{2}}
$$

are used. For instructions how to derive the expressions for the $\nabla$-operator, see Aris [1962].

\subsection{Scaling Analysis}

The coordinates $\{\nu, \alpha, z\}$ and the time $t$ are scaled to typical scales for laser fusion cutting

$$
\nu=d_{m} \nu^{\prime}, \quad \alpha=\alpha^{\prime}, \quad z=d z^{\prime}, \quad r=w_{0} r^{\prime}, \quad t=\frac{d_{m}}{v_{0}} \tau
$$

[Wandera and Kujanpaa, 2010]. The scaled velocities $\mathbf{u}=\left\{u_{\nu}, u_{\alpha}, u_{z}\right\}$ are given by

$$
v_{\nu}=v_{0} u_{\nu}, \quad v_{\alpha}=\frac{w_{0}}{d_{m}} v_{0} u_{\alpha}, \quad v_{z}=\frac{d}{d_{m}} v_{0} u_{z} .
$$

The typical temperature $\theta$ maps the temperature $T$ to the interval between ambient temperature $T_{a}$ and melting temperature $T_{m}$ and the scaled pressure $\Pi$ is given by

$$
T=\left(T_{m}-T_{a}\right) \theta+T_{a}, \quad p=\frac{d^{2} \varrho \nu_{l} v_{0}}{d_{m}^{3}} \Pi
$$


U. Jansen et al., Boundary layer approximation for melt film dynamics in laser fusion cutting

The hierarchy of the spatial dimensions is depicted by the small parameters $\gamma, \delta$ and $\epsilon$

$$
\epsilon=\frac{d_{m}}{d} \ll \delta=\frac{d_{m}}{w_{0}} \wedge \epsilon \ll \gamma=\frac{w_{0}}{d} \wedge \mathcal{O}(\delta)=\mathcal{O}(\gamma)
$$

For an exemplary cutting task in stainless steel the scales are $d_{m}=30 \mu \mathrm{m}, w_{0}=300 \mu \mathrm{m}$ and $d=6 \mathrm{~mm}$. With the Reynolds number $\operatorname{Re}=v_{0} d \nu_{l}^{-1}=224$ follows:

$$
\epsilon=0.005, \quad \delta=0.1, \quad \gamma=0.05
$$

with the relations

$$
\epsilon=\gamma \delta, \quad \delta^{2}=\epsilon \frac{\delta}{\gamma}=\mathcal{O}(\epsilon), \quad \gamma^{2}=\epsilon \frac{\gamma}{\delta}=\mathcal{O}(\epsilon) \quad \text { and } \quad \epsilon \operatorname{Re}=\operatorname{Re}^{*}=\mathcal{O}(1)
$$

For the Lamé coefficients (9) follows

$$
h_{\nu}=h_{\nu}^{\prime}, \quad h_{\alpha}=w_{0} h_{\alpha}^{\prime}, \quad h_{z}=h_{z}^{\prime} .
$$

The primed quantities $\left[\nu^{\prime}, \alpha^{\prime}, z^{\prime}, r_{m}^{\prime}, h_{\nu, \alpha, z}^{\prime}\right]$ are now written as non-primed quantities to ensure better readability. All following quantities are written as non-primed and denote the scaled (primed) quantities.

The derivatives of $h_{z}$ with respect to $\alpha$ and $z$ contain small parameters $\gamma^{2}=\epsilon \gamma / \delta$

$$
\partial_{\nu} h_{z}=0, \partial_{\alpha} h_{z}=\gamma^{2} \frac{\partial_{z} r_{m} \partial_{\alpha}\left(\partial_{z} r_{m}\right)}{h_{z}}, \partial_{z} h_{z}=\gamma^{2} \frac{\partial_{z} r_{m} \partial_{z}^{2} r_{m}}{h_{z}},
$$

and the derivative $h_{\alpha}$ with respect to $\nu$ contains the small parameter $\delta=\epsilon / \gamma$ :

$$
\partial_{\nu} h_{\alpha}=\epsilon \frac{1}{\gamma} \frac{\delta \nu-r_{m}}{h_{\alpha}}
$$

\subsection{Perturbation expansion}

Transforming equations (2) for mass balance, momentum balance and energy to conformal coordinates and by applying the scales denoted in (10), the resulting equations can be ordered by powers of the small parameter $\epsilon$. The resulting structure of the equations is

$$
\begin{aligned}
\partial \nu \Pi & =\epsilon^{2} f_{\nu 2}+\epsilon^{3} f_{\nu 3}+\epsilon^{4} f_{\nu 4}+\epsilon^{5} f_{\nu 5} & & \operatorname{Momentum}(\nu) \\
\frac{1}{h_{\alpha}} \partial_{\alpha} \Pi & =\epsilon^{1} f_{\alpha 1}+\epsilon^{2} f_{\alpha 2}+\epsilon^{3} f_{\alpha 3}+\epsilon^{4} f_{\alpha 4} & & \operatorname{Momentum}(\alpha) \\
\frac{1}{h_{z}} \partial_{z} \Pi & =\epsilon^{0} f_{z 0}+\epsilon^{1} f_{z 1}+\epsilon^{2} f_{z 2}+\epsilon^{3} f_{z 3} & & \operatorname{Momentum}(z) \\
0 & =\epsilon^{0} f_{\nabla 0}+\epsilon^{1} f_{\nabla 1}+\epsilon^{2} f_{\nabla 2} & & \operatorname{MasS} \\
0 & =\epsilon^{0} f_{\theta 0}+\epsilon^{1} f_{\theta 1}+\epsilon^{2} f_{\theta 2} & & \text { Energy }
\end{aligned}
$$

where $f_{\{\nu, \alpha, z, \nabla, \theta\}}$ denote the terms for the corresponding equation and the power of the small parameter $\epsilon$. The hierarchy of the spatial dimensions is indicated by the $\epsilon$-order of the leading term of the momentum balance. The $z$-derivative of the pressure $\Pi$ starts with $\mathcal{O}(1)$, the $\alpha$-derivative with $\mathcal{O}(\epsilon)$ and the $\nu$-derivative with $\mathcal{O}\left(\epsilon^{2}\right)$. This hierarchy allows a solution using a power series of the small parameter $\epsilon$ for the quantities $\theta, \Pi$ and $\mathbf{u}$

$$
\begin{aligned}
\theta & =\theta_{0}+\epsilon \theta_{1}+\ldots+\epsilon^{N} \theta_{N} \\
\Pi & =\Pi_{0}+\epsilon \Pi_{1}+\ldots+\epsilon^{N} \Pi_{N} \\
\mathbf{u} & =\mathbf{u}_{0}+\epsilon \mathbf{u}_{1}+\ldots+\epsilon^{N} \mathbf{u}_{N}
\end{aligned}
$$

By substituting (20) into (19), the equations can be solved in ascending orders of $\epsilon$ using the solution of preceding orders where these are required. For the order $\mathcal{O}\left(\epsilon^{0}\right)$ follows:

$$
\partial_{\nu} \Pi_{0}=0, \quad \frac{1}{h_{\alpha}} \partial_{\alpha} \Pi_{0}=0, \quad \frac{1}{h_{z}} \partial_{z} \Pi_{0}=f_{z 0}\left(\mathbf{u}_{0}\right), \quad 0=f_{\nabla 0}\left(\mathbf{u}_{0}\right)
$$


and for the order $\mathcal{O}\left(\epsilon^{1}\right)$ :

$$
\partial_{\nu} \Pi_{1}=0, \frac{1}{h_{\alpha}} \partial_{\alpha} \Pi_{1}=f_{\alpha 1}\left(\mathbf{u}_{0}\right), \frac{1}{h_{z}} \partial_{z} \Pi_{1}=f_{z 0}\left(\mathbf{u}_{1}\right)+f_{z 1}\left(\mathbf{u}_{0}\right), 0=f_{\nabla 0}\left(\mathbf{u}_{1}\right)+f_{\nabla 1}\left(\mathbf{u}_{0}\right)
$$

The expansions for higher orders of $\epsilon$ are carried out analogous. The writing $f_{\{\nu, \alpha, z, \nabla\} n}\left(\mathbf{u}_{m}\right)$ denotes that the $m$ th order of the expansion (20) of the velocity $\mathbf{u}$ is used for the $n$th term of the perturbation series (19). The presented expansion shows that for every order of the small parameter $\epsilon$ four equations for four unknowns have to be solved. For orders of $\epsilon$ greater than zero, solutions of preceding orders have to be added as constant contribution to the equation of the current order. The fifth equation for the temperature $\theta$ can be solved independently after the equations for momentum and mass balance are solved. The given formulation of the original equations (2) is exact as long as the perturbation series is evaluated for all orders of $\epsilon$.

\subsection{Integral Model}

To reduce the dimension of the differential equation (19), the momentum method [Kubota and Dewey, 1964] is used. The equations (19) are integrated in radial $(\nu)$ direction, using a quadratic approximation for the velocities $u_{\alpha}$ and $u_{z}$. The resulting integrated quantities $m_{\alpha}$ and $m_{z}$ describe the mass flow in azimuthal and axial directions for a melt film thickness $h$ given by the kinematic boundary condition (7). A steady state solution for low orders of $\epsilon$ is depicted in Figure 5. Note: To obtain integrable expressions for the leading orders of the functions $f_{\{\nu, \alpha, z, \nabla\} n}$, the expression $h_{\alpha}^{-1}$ has to be expanded into a Taylor series at $\nu=0$. The derivatives $\partial_{\nu}^{n} h_{\alpha}^{-1}$ contain small parameters which can be assigned to higher $\epsilon$-orders. Also the limiting behavior for values of the melt film thickness $h<\epsilon$ has to be considered.

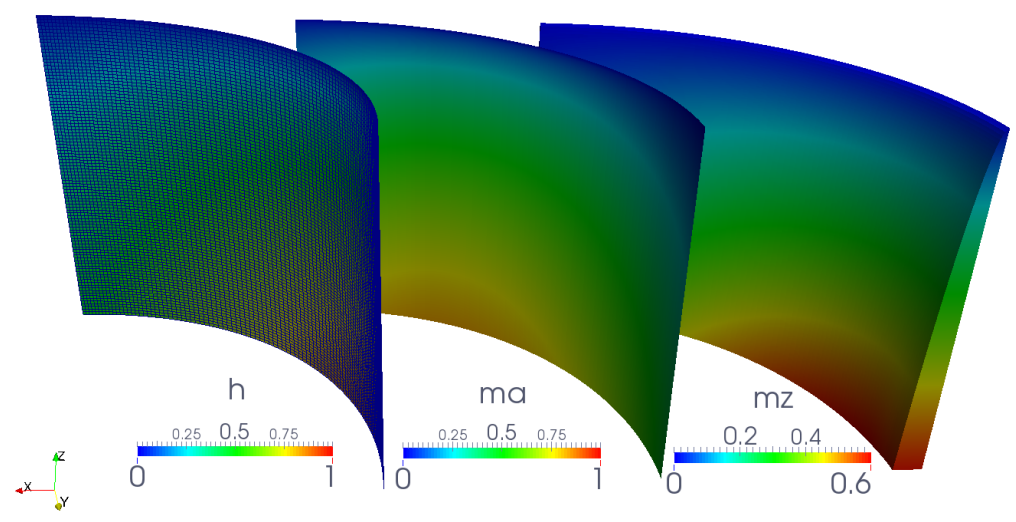

Figure 5. Scaled stationary solution for melt film thickness $h$, azimuthal mass flux $m_{\alpha}$ and axial mass flux $m_{z}$ for the lowest order of the small parameter $\epsilon$ in 3D space. The solution for the mass flux $m_{\alpha}$ is warped by the melt film thickness $h$ to illustrate the shape of the melt film. The computational mesh is indicated in the solution for the melt film thickness $h$.

\section{Discussion}

A method to reduce the computational effort in accurate solving of the incompressible Navier Stokes equations for a thin melt film layer in laser fusion cutting is presented in this paper. The mathematical task is transformed to conformal coordinates of the melt film, which allow a scaling analysis in the three spatial dimensions. The formulation of the task in conformal coordinates allows a dimension reduction by one using the momentum method. The remaining set of equations can be solved numerically on a rectangular mesh of the azimuthal $(\alpha)$ and axial $(z)$ coordinates using a finite volume approach for the integrated mass fluxes $m_{\alpha}$ and $m_{z}$ and the melt film thickness $h$. The strong advantage compared to direct numerical solutions of the melt film is the reduced number of degrees of freedom due to the quadratic approach for the velocity field in the melt film and the combined solving of the melt film thickness along with the mass flux. The expansion of the equations by 
the small parameter $\epsilon$ allows a iterative solution and neglecting higher orders if applicable to safe computation time. A steady state solution for the lowest order of the small parameter $\epsilon$ provides reasonable results, the dynamic calculation and the consideration of higher orders is currently being implemented. The expected reduction in computational costs compared to classical finite volume solvers can be used to increase the spatial and temporal accuracy of the solution to describe effects like the separation of ligaments from the melt film and the propagation of perturbations.

\section{ACKNOWLeDgments}

All presented investigations are conducted in the context of the Collaborative Research Centre SFB1120 "Precision Melt Engineering" at RWTH Aachen University and funded by the German Research Foundation (DFG). For the sponsorship and support we wish to express our sincere gratitude.

\section{REFERENCES}

Amara, E. H., K. Kheloufi, T. Tamsaout, R. Fabbro, and K. Hirano (2015, Jun). Numerical investigations on high-power laser cutting of metals. Applied Physics A 119(4), 1245-1260.

Aris, R. (1962). Vectors, tensors and the basic equations of fluid mechanics. Dover Publications, Inc. New York.

Arntz, D., D. Petring, U. Jansen, and R. Poprawe (2017). Advanced trim-cut technique to visualize melt flow dynamics inside laser cutting kerfs. Journal of Laser Applications 29(2), 022213.

Kato, T. (1995). Perturbation Theory for Linear Operators (2 ed.). Springer-Verlag Berlin Heidelberg.

Kheloufi, K., E. H. Amara, and A. Benzaoui (2015, Jun). Numerical simulation of transient three-dimensional temperature and kerf formation in laser fusion cutting. Journal of Heat Transfer 137(11), 112101/1$112101 / 9$.

Kohl, S. and M. Schmidt (2013, Oct). Numerical Analysis of the Influence of Beam Characteristics onto the Process Dynamics during Laser Cutting. In The 32nd International Congress on Applications of Lasers \& Electro-Optics (ICALEO) 2013. Omnipress.

Kubota, T. and C. F. Dewey (1964, Apr). Momentum integral methods for the laminar free shear layer. AIAA Journal 2(4), 625-629.

Langtangen, H. P., K.-A. Mardal, and R. Winther (2002). Numerical methods for incompressible viscous flow. Advances in Water Resources 25(8), 1125-1146.

Lopez-Sandoval, E. and A. Mello (2012). Series solution of non linear partial differential equations from physical mathematical. CoRR abs/1206.2346.

Mahrle, A. and E. Beyer (2009). Theoretical aspects of fibre laser cutting. Journal of Physics D: Applied Physics 42(17), 175507.

Otto, A. and M. Schmidt (2010). Towards a universal numerical simulation model for laser material processing. Physics Procedia 5, 35 - 46. Laser Assisted Net Shape Engineering 6, Proceedings of the LANE 2010.

Poprawe, R., W. Schulz, and R. Schmitt (2010). Hydrodynamics of material removal by melt expulsion: Perspectives of laser cutting and drilling. Physics Procedia 5, 1-18. Laser Assisted Net Shape Engineering 6, Proceedings of the LANE 2010, Part 1.

Schulz, W., V. Kostrykin, M. Nießen, J. Michel, D. Petring, E. W. Kreutz, and R. Poprawe (1999). Dynamics of ripple formation and melt flow in laser beam cutting. Journal of Physics D: Applied Physics 32(11), 1219.

Schulz, W., M. Nießen, U. Eppelt, and K. Kowalick (2009). Simulation of Laser Cutting, pp. 21-69. Dordrecht: Springer Netherlands.

Vossen, G. and J. Schüttler (2012). Mathematical modelling and stability analysis for laser cutting. Mathematical and Computer Modelling of Dynamical Systems 18(4), 439-463.

Wandera, C. and V. Kujanpaa (2010). Characterization of the melt removal rate in laser cutting of thick-section stainless steel. Journal of Laser Applications 22(2), 62-70. 